\title{
Autor index
}

\author{
Abbasov M.A. 181 \\ Abdukarimov Sh. 38 \\ Abekova A.M. 17 \\ Abugalieva A. 79 \\ Abugalieva S. 23, 79 \\ Adonina I.G. 18, 52, 215, 238 \\ Afanasenko O.S. 19, 109, 137, 187 \\ Afonin A.M. 131 \\ Afonnikov D.A. 78, 179, 203 \\ Agaeva E.V. 51 \\ Ageev A.Yu. 20 \\ Ageeva E.V. 21 \\ Akparov Z.I. 181 \\ Alekseev V.Y. 22, 189 \\ Aliyeva A.J. 181 \\ Almerekova S. 79 \\ Amalova A. 23 \\ Amangeldiyeva A.A. 17 \\ Anapiyaev B. 144 \\ Anashkina A.A. 24 \\ Andreeva E.A. 222 \\ Anisimova O.K. 25 \\ Antipin M.I. 195 \\ Antonov A.A. 117 \\ Antonova O.Yu. 77, 176 \\ Aparina V.A. 26, 43, 174 \\ Arbuzova G. 144 \\ Artyushin I.V. 195 \\ Aseeva T.A. 174 \\ Askhadullin Damir F. 27, 28 \\ Askhadullin Danil F. 27, 28 \\ Aubakirova K.P. 88 \\ Azarova T.S. 35 \\ Babina D.D. 106 \\ Babisekova D.I. 17 \\ Babkenov A. 23 \\ Babkenova S. 23 \\ Badaeva E.D. 29, 52 \\ Bagavieva E.Z. 28 \\ Balashova I.T. 30 \\ Baltin S.M. 31, 224 \\ Bankin M. 208 \\ Baranova O.A. 32, 135, 136, 137 \\ Barbitob Y.A. 87 \\ Bastaubayeva Sh.O. 17 \\ Baygutov M.Zh. 188 \\ Bazhenov M.S. 53, 213
}

Bazylova T.A. 17

Bebykina I.V. 52

Bektayev R.T. 100

Bekhtold N.P. 33, 78

Belan I.A. 34, 170

Belenikin M.S. 195

Belimov A.A. 35

Belkov V.A. 75

Belovezhets L.A. 172

Bemova V.D. 36

Ben C. 48

Berezhnaya A.A. 37, 115

Bespalova L.A. 51, 213

Birukhov V.V. 40

Bishop-von Wettberg E. 20

Blokhina N.P. 34

Bobokhujaev Sh.U. 38, 193

Bogdanova O.V. 158

Boldakov D.M. 51

Boldyrev S. 90

Bome N.A. 39, 227

Bondar E.I. 40

Bondarenko N.P. 134

Bondarevich E.B. 57

Boris K.V. 220, 221

Borisenko N.V. 64

Borovaya S.A. 41

Borovskii G.B. 42, 85

Boulygina E.S. 81, 207

Bovet L. 101

Boyko N.I. 26, 43, 174

Bragina M.K. 114, 115, 196

Budhagatapalli N. 94

Bukin Yu.S. 160

Bulavin I.V. 44

Buriev Z. 38

Burkhanova G.F. 22, 45, 161, 189, 201, 230

Bychkov I.A. 46

Börner A. 47

Büchner H. 50, 194

Chalaya N.A. 62, 119

Chamas S. 50, 62, 124, 194

Changalidi A.I. 87

Chaouachi M. 48

Chasov A.V. 164

Chaturvedi D. 198

Chekunova E.M. 49 
Cherepanova E.A. 45, 189

Chernook A.G. 53

Chernova A. 90

Chernyak E.I. 120

Chilimova I.V. 227

Chirva O.V. 91

Chubukova O.V. 228

Chumakov M.I. 153

Chursin A.S. 202

Clapco S. 58

Daghma D.E.S. 50, 194

Danilova M.N. 55, 145

Davoyan E.R. 51, 52

Davoyan R.O. 51, 52

Davydenko O. 206

De Palo D. 101

Demurin Y. 90

Dergilev A.I. 24, 165

Divashuk M.G. 53, 113, 213

Djébali N. 48

Dmitriev A.A. 157

Dobrovolskaya O.B. 96, 165

Dodueva I.E. 72, 190, 216

Dolgikh V. 54

Dolgov S. 70, 151, 213

Dolinny Y.Y. 227

Domrachev D.V. 62, 120, 124

Doroshenko A.S. 55, 145

Doroshkov A.V. 122

Druzhin A.E. 32

Duarte G.T. 232

Dubina E.V. 56, 125

Dubovets N.I. 57, 166

Duca M. 58

Dudnikov M. 59, 113

Duk M. 60

Dushkin V.A. 117

Dvorianinova E.M. 157

Dvornikova K.A. 138

Dyachenko E.A. 156

Efimov V.M. 187

Efimova M.V. 155

Efremov G.I. 61

Efremova I.G. 111

Egorova A.A. 62, 63, 71, 80, 119, 191

Elatskov Yu. 210

Elatskova A. 210

Elkahoui S. 48

Elkonin L.A. 64

Emirsaliev A.O. 152, 223

Enzekrey E.S. 173
Epifanov R. 65

Ermakovich A. 206

Ermolenko O.I. 89

Evtushenko E.V. 66, 76

Fadeev V.V. 153

Fakhranurova L.I. 103

Fedorova O.V. 171

Fedoseeva I.V. 42

Fedotova O.A. 67, 85

Fedotovskaya V. 68

Fedyaev V. 229

Fedyaeva A.V. 42

Feranchuk S.I. 40

Fesenko A.N. 141

Filyushin M.A. 25, 69

Firsov A. 70

Fomin I.N. 71

Gainullin N. 135

Galibina N.A. 91

Gall N.R. 132

Galushko A.S. 132

Gamburg K.Z. 85

Gancheva M.S. 72, 127, 142, 190

Ganenko T.V. 159

Garapov D.S. 73

Garibyan Ts.S. 74

Garkusha S.V. 56

Garnik E.Yu. 75

Gatzkaya S.S. 66, 76

Gavrilenko T. 77

Genaev M.A. 65, 78

Genievskaya Y. 79

Gentzbittel L. 48

Gerashchenkov G.A. 64

Gerasimova S.V. 62, 63, 71, 80, 119, 120, 123,124

Gladysheva-Azgari M.V. 81, 207

Glagoleva A.Yu. 82, 130, 231

Goepfert S. 101

Gorbenko I.V. 83

Gorbunova A.O. 129, 236

Gordon M.L. 131

Gorislavets S.M. 233

Gorshkova T.A. 84

Goryunov D. 90

Goryunova S. 90

Grabelnych O.I. 67, 85

Graskova I.A. 169

Gretsova M. 86

Gribchenko E.S. 131

Grigoreva E.A. 87, 143, 177 
Grigoriev Y.N. 130

Grishko E.O. 66

Gritsenko D.A. 88, 178

Gruzdev I.V. 89, 118

Gubaev R. 90

Guchenko S.S. 97

Gulayeva E.N. 91

Gultyaeva E.I. 32, 78

Gurina A.A. 92

Guro P.V. 35

Gusev Yu.S. 153

Gvaramiya S. 93, 113, 147, 162

Hackauf B. 222

Hansson M. 204

Hertig C. 62, 63, 80, 94, 120, 123

Hiekel S. 62, 63, 80, 94

Ibragimova S. 62, 95, 191

Ignatov A.N. 96

Ilyushko M.V. 97

Ivanov N.V. 101

Ivanova E.A. 98

Ivanova N.N. 44

Ivanova Yu.N. 99

Jacobi A. 94

Jallouli S. 48

Junker A. 94

Kalendar R.N. 100

Kaminski K.P. 101

Kamionskaya A.M. 156

Kanapin A. 60, 86, 208

Kapustyanchik S.Yu. 102

Karataeva T.A. 103

Karepova M.S. 172

Karlov G.I. 53, 113, 213

Karzhaev D.S. 87, 104, 177

Kasianov A.S. 105, 116, 141

Katyshev A.I. 42

Kazakov E.I. 106

Kazakova E.A. 106

Kelbin V.N. 107, 136

Kenzhegulov O.A. 64

Khabibrakhmanova V.R. 182

Khaitovich P. 90

Khakimova L.R. 228

Khandy M.T. 108

Khatefov E.B. 69

Khiutti A.V. 19, 109

Khlestkina E.K. 63, 80, 82, 120, 123, 130, $148,187,210,231$
Khudokormova J.N. 51

Khusainova I.I. 28

Khusnutdinov E. 110

Khutsishvili S.S. 159

Kibalnik O.P. 111

Kilian B. 29

Kingsly J.N.B. 112

Kirichenko K. 144

Kirov I. 59, 93, 113, 118, 147, 162

Kiseleva A.A. 37, 114, 115, 121, 140

Kiseleva M.I. 136

Klementyeva A. 151, 213

Klepikova A.V. 116, 141

Klimenko I.A. 117

Klykov A.G. 41

Klyushin A.G. 108

Kochetov A.V. 62, 80, 95, 115, 120, 191, 196, 203

Kochieva E.Z. 61, 69, 156

Koeppel I. 62, 63, 80

Kolenkov M.A. 89, 118

Kolganova E. 113

Kolokolova N.N. 39

Kolomiets T.M. 136

Koloshina K.A. 71, 119

Kolosovskaya E.V. 80, 120, 123

Kolozhvari A.E. 115, 121

Komakhin R. 93, 113

Kondakova M.A. 42

Konorov E.A. 195

Konstantinov D.K. 122

Konstantinov Yu.M. 75, 83

Konstantinov Z.S. 74

Konusbekov K.T. 17

Korotaeva N.E. 42

Korotkova A.M. 80, 120, 123

Korsukova A.V. 85

Kostina N.E. 124

Kostylev P.I. 125

Kovalenko N. 135

Kovalenko T. 90

Kovaleva O.N. 82

Kovlagina M. 59

Kovtun A.S. 105

Kozlov K.N. 20

Kradetskaya O.O. 227

Krasnoperova E.Y. 126, 224

Krinitsina A.A. 195

Kriukova K.I. 127

Krivenko O. 152

Kroupin P. 213

Krutovsky K.V. 40, 128

Kryukov A.A. 129, 236 
Kudriashov A.A. 237

Kudryakova N.V. 46

Kudryavtsev A.M. 221

Kudryavtseva L.P. 157

Kudryavtseva Y.A. 172

Kukoeva T.V. 130, 204, 231

Kukoleva S.S. 111

Kulaeva O.A. 131

Kuleshova O.N. 152, 223

Kuleshova T.E. 132

Kulikova D.K. 24

Kumar D. 198

Kumlehn J. 50, 62, 63, 80, 94, 120, 123, 124, 133, 185, 194

Kuptsov S.V. 195

Kushnirenko I.Yu. 134

Kusnetsov V.V. 46

Kuvaeva D.D. 199

Kuzmin D.A. 40

Kuzmin O.G. 202

Kuznetsova K.A. 216

Lang G. 101

Laparra H. 101

Lapidus A. 183

Lapochkina I. 135

Laprina Yu.V. 136

Larichev K.T. 196

Lashina N.M. 19, 109, 137, 187

Lebedeva M.A. 138, 192

Lee C.-R. 20

Leksin I.Y. 139, 146, 164, 182

Leonova I.N. 21, 115, 140, 166

Lesnyak S.A. 56

Levites E.V. 235

Levitsky V. 54

Liaudansky A. 206

Likhovskoi V.V. 177, 233

Litvinov D.Y. 53

Logacheva M.D. 86, 141, 195

Losev M.R. 142

Lushchay E.A. 233

Lutova L.A. 31, 72, 126, 127, 138, 142, 190 , 192, 216, 224, 234, 237,

Luzin A.N. 24, 96

Lykhina A.V. 124

Lytkin K.F. 143

Lyubushkina I. 144

Makamov A. 38

Makarenko M.S. 141

Makarova T. 77

Maksimov I.V. 22, 45, 161, 189, 201
Malyukova A.M. 55, 145

Marchenkova L. 135

Markova Y.A. 172

Martea R. 58

Marthe C. 94

Martynova E. 90

Marzouk T. 48

Matsushita Y. 19, 109

Matveeva T.V. 36

Maulenbay A.D. 188

Mazilov S.I. 153

Mazina A.B. 146, 164

Mehdiyeva S.P. 18, 181, 238

Melnikova N.V. 157

Merkulov P. 93, 113, 147, 162

Mikhailova A.S. 148

Mikhaylova E. 110

Mikhaylova Yu.V. 129, 149, 236

Mikov D.S. 51

Milyukova N.A. 173

Minasbekyan L.A. 150

Minibayeva F.V. 139, 146, 164, 182

Mironenko N.V. 19, 109, 137

Miroshnichenko D. 151, 213

Mirzakhmedov M. 50, 194

Mishin A.S. 103

Mitiouchkina T.Y. 70, 103

Mitrofanova I.V. 44, 81, 152, 207, 220, 223

Mitrofanova O.P. 176

Moiseev A.S. 106

Moiseeva E.M. 153

Moldavanov A. 154

Morgounov A.I. 202

Morozov S.V. 120

Morozova E.V. 26, 174

Mukhina Y.V. 34

Murgan O.K. 155

Mutu A. 58

Nezhdanova A.V. 156

Nikitin P.A. 195

Nikolski M. 212

Nosov A.M. 108

Novakovskiy R.O. 157

Novikova A.A. 158

Novokazi F. 137

Nozhkina O.A. 159, 169

Nurminsky V.N. 160

Nuzhdin S.V. 20, 86

Nuzhnaya T.V. 161, 230

Omarov M. 93, 113, 147, 162

Omelchenko D.O. 141, 195 
Omelyanchuk N.A. 163

Onele A.O. 164

Oreshkova N.V. 40

Orlov Y.L. 24, 96, 165

Orlova E.A. 33, 78, 102

Orlovskaya O.A. 166

Oshchepkov D. 54

Osipova S.V. 180, 205

Otto I. 123

Ozherelev P. 118

Ozolina N.V. 160

Pakina E.N. 96

Panfilova M. 110

Panin V.M. 64

Panova G.G. 132

Park M.E. 219

Pavlichenko V.V. 167

Pendinen G. 77

Penin A.A. 105, 116, 141

Peretyagina T. 90

Perfil'ev R.N. 168

Perfileva A.I. 159, 169

Permyakov A.V. 180, 205

Permyakova M.D. 180, 205

Pershina L.A. 34, 170

Petrash N.V. 171

Petrushin I.S. 83, 172

Pirsikov A.S. 173

Piskarev V.V. 26, 43, 174, 218

Podlutskii M.S. 106, 232

Podobed M.Yu. 106

Poliushkevich L.O. 142

Polyakova E.A. 67, 85

Polyakova M. 144

Pomortsev A. 144

Ponomarev S.N. 175

Ponomareva M.L. 175

Porotnikov I.V.176

Port A. 58

Poteshkina A.A. 174

Potokina E.K. 87, 143, 177, 233

Pototskaya I.V. 202

Potsenkovskaia E.A. 224

Poursarebani N. 94

Povkhova L.V. 157

Pozharskiy A.S. 88, 178

Pravdina O.Yu. 216

Predeus A.V. 216

Pronozin A. 179

Protopopova M.V. 167

Pshenichnikova T.A. 180, 200, 205

Pundir S. 198
Pushin A. 70

Pushkova E.N. 157

Rahimov R.G. 181

Rassabina A.E. 182

Rayko M. 183

Rekoslavskaya N.I. 184, 209

Rezaeva B. 185

Rikhvanov E.G. 42

Risovannaya V.I. 233

Rodionov A.V. 236

Rogozina E.V. 62, 119

Romanov D.V. 186

Romanova A.V. 62, 95, 124

Romanova O.I. 141

Romanyuk D.A. 131

Romashova M.V. 97

Rosseeva L.P. 34, 170

Rozanova I.V. 137, 187

Rozhmina T.A. 60, 157, 208

Rozhnova N.A. 64

Rsaliyev A.S. 188

Ruban M.G. 56

Rumyantsev S.D. 189, 230

Rutkovskaya E.A. 190

Saboiev I. 62, 95, 191

Saburov V.O. 106

Sadikova D.S. 138, 192

Sadovsky M. 68

Safronova V.I. 35

Salina E.A. 18, 21, 37, 52, 62, 95, 107, 114, $115,121,140,151,168,191,196,199$, 215,238

Salyaev R.K. 184, 209

Samoilov A.E. 195

Samsonova A. 60, 86, 208

Samsonova M.G. 20, 60, 86, 208

Sanamyan M.F. 38, 193

Sarsenova S.Kh. 64

Satpathy P. 50, 194

Sauchyn D.G. 42

Sazanova A.L. 35

Schafleitner R. 20

Schelkunov M.I. 141

Scherbakov A. 86

Schnurbusch T. 94

Scobeyeva V.A. 195

Sekste E.A. 35

Sergeeva E.M. 196

Shaloiko L. 70

Shamanin V.P. 107, 202

Shaposhnikov A.I. 35 
Sharapova L.S. 197

Sharko F.S. 81, 207

Sharma Shailendra 198

Sharma Shiveta 198

Sharov V.V. 40

Shchennikova A.V. 61, 69, 156

Shcherban A.B. 18, 168, 199

Shchukina L.V. 200

Shein M.Yu. 22, 161, 201

Shepelev S.S. 202

Shishova M.F. 149, 236

Shlyavas A.V. 221

Shmakov N.A. 80, 120, 203, 231

Shoeva O.Y. 63, 80, 82, 130, 204, 230, 231

Shreyder E.R. 134

Shtark O.Y. 131

Shyshlova-Sokolovskaya A.M. 42

Sibikeev S.N. 32

Sierro N. 101

Siksin S.V. 173

Silkova O.G. 99

Simonov A.V. 180, 205

Singh V.K. 198

Siniauskaya M. 206

Sirota S.M. 30

Skolotneva E.S. 78, 99, 107, 136

Slobodova N.V. 81, 207

Slugina M.A. 156, 225

Smirnova O.G. 180, 205

Smykov A.V. 220

Sochalova L.P. 174

Sofronova A.K. 108

Sokolkova A. 208

Sokolova L.M. 30

Sokolova N. 144

Sokolyuk A.V. 57

Sokornova S. 183

Solodyankin P.A. 49

Solovey L.A. 57, 166

Soloviev A.A. 59, 89, 113, 118

Solovyeva A.I. 214

Sorokan A. 45

Spaselnikova A.V. 124

Speranskaya A.S. 195

Stasyuk A.I. 99, 115, 140

Stepanov A.V. 85

Stepanov I.V. 73

Stepanova A.Y. 214

Stolbikov A.S. 184, 209

Strygina K.V. 63, 80, 130, 148, 210

Sukhareva S. 110

Sukhomlinov V.Yu. 26, 43, 174, 211

Sukhorukov G. 212
Surkova S. 86, 208

Sycheva E.A. 57

Syrova D.S. 35

Tarasenko V.I. 75

Tarelkina T.V. 91

Tazutdinova M.R. 28

Tekhanovich G. 210

Tetyannikov N.V. 39

Thieme R. 77

Tikhonovich I.A. 131

Timerbaev V. 151, 213

Timergalina L. 229

Timin O.Y. 214

Timina O.O. 214

Timonova E.M. 215

Ting C.-T. 20

Tkachenko A.A. 87, 216, 217

Tomilin M.A. 124

Tomilova S.V. 108

Toropova E.Yu. 211, 218

Tretyakova I.N. 219

Trifonova A.A. 220, 221

Trubacheeva N.V. 34, 170

Trunov R.I. 218

Tsvetkova N.V. 222

Tsygankova S.V. 81, 207

Tsyupka V.A. 152, 220, 223

Turuspekov Y. 23, 79

Tvorogova V.E. 31, 126, 216, 224, 234, 237

Tyapkina D.Y. 225

Ubogoeva E.V. 163

Udalova O.R. 132

Ukolova I.V. 226

Ulianich P.S. 87, 177, 217

Urbanovich O.Yu. 42

Utebayev M.U. 227

Vainstein A. 70

Vardevanyan P.O. 150

Vasiliev G.V. 203

Vasilova N.Z. 27, 28

Vasylyk I.A. 177, 233

Vershinin A.V. 66, 76

Vershinina Z.R. 228

Veselov D. 229

Veselova S.V. 22, 161, 189, 230

Vikhorev A.V. 63, 80, 120, 231

Vlasova A.A. 75

Voinikov V.K. 144, 167

Volkov V.A. 87, 177

Volkova P.Yu. 106, 232 
Volodin V.A. 233

Volynkin V.A. 177, 233

Voylokov A.V. 222

Vysotskaya L. 229

Weisfeld L.I. 39, 227

Yakovleva D.V. 224, 234

Yanyshevskaya A. 210

Yashenkova Ya.S. 138

Yerzhebayeva R.S. 17

Yudina R.S. 235

Yurina N.P. 197

Yurkov A.P. 129, 236

Yuzikhin O.S. 35

Zabanova N.S. 85

Zakharova E.V. 74
Zakhrabekova S. 204

Zemlyanskaya E.V. 54, 163

Zenkina K.V. 174

Zhdanova I.V. 223

Zhukov V.A. 131

Zhurbenko P.M. 236

Zinchenco A.N. 52

Zlydneva N.S. 224, 237

Zorin E.A. 131

Zorina M.V. 18, 238

Zubanova Yu.S. 51, 52

Zubkovich A.A. 137

Zuev E.V. 28, 43

Zykin P.A. 222

Özkan H. 29 